\title{
An experimental investigation into the effect of breakfast consumption on energy intake and expenditure
}

\author{
L. Halsey, J. Huber, T. Low, C. Ibeawuchi and S. Reeves \\ School of Human and Life Sciences, Roehampton University, Holybourne Avenue, London SW15 4JD, UK
}

Breakfasting frequency has commonly been reported to be inversely associated with BMI. The causal factor is often assumed to be an increased energy intake later in the day in breakfast skippers ${ }^{(1)}$. Alternatively, it has been suggested that daily breakfast eaters could be more physically active than breakfast skippers ${ }^{(2)}$. However, there has been little research focusing on differences in energy expenditure between these two groups. Heart-rate monitors and pedometers are frequently used to assess physical activity in field conditions ${ }^{(3)}$ and can also be used to assess energy expenditure when an individual calibration between heart rate and energy expenditure is conducted. The present research aimed to evaluate and compare energy intake, energy expenditure and physical activity using an experimental design in which the consumption of breakfast or otherwise was controlled.

Twenty females (aged 19-25 years) wore a heart-rate monitor (Polar; Polar Electro, Oulu, Finland) and a pedometer (Yamax, Yamasa Tokei Keiki Co. Ltd, Tokyo, Japan) for five consecutive days when they were provided with breakfast and for $5 \mathrm{~d}$ when they were not permitted to eat breakfast. A cross-over design was applied and in both conditions further food could not be consumed until midday. The participants were asked to complete two $3 \mathrm{~d}$ food diaries, one during each phase of the study. Food diaries were analysed using Dietplan6 (Forestfield Software, Horsham, West Sussex, UK) and Excel. The heart rate of participants was individually calibrated with rate of $\mathrm{O}_{2}$ consumption via respirometry (Jaegar Oxycon; VIASYS Healthcare GmbH, Hoechberg, Germany) to enable the estimation of energy expenditure from the heart-rate-monitor data.

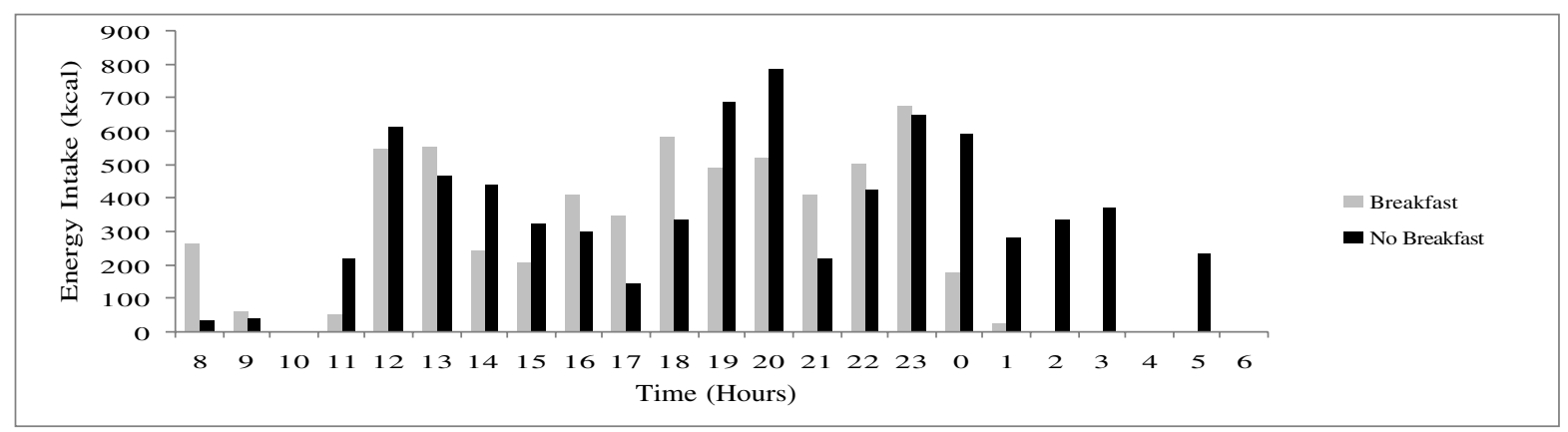

Repeated measures ANOVA revealed an interaction between energy intake and time of day with a significant $(P<0.05)$ increase in the amounts consumed after midnight on non-breakfast days, as shown in the Figure. There was no significant difference in total energy intake (8925 (SD 2552) kJ (2133 (SD 610) kcal)/d v. 8577 (SD 3268) kJ (2050 (SD 781) kcal)/d), pedometer readings (steps per d; 5641 (SD 610) v. 6406 (SD 4115)), or mean heart rate (beats per min; 85 (SD 9) v. 84 (SD 9)) between breakfast and non-breakfast days. It would appear that, although eating breakfast or not eating breakfast did not affect the energy consumed or energy expended, it did affect the timing of food intake, with participants eating later on days when they had no breakfast. These changes in eating pattern could be a reactive mechanism to compensate for the lack of breakfast that morning or an anticipatory strategy to alleviate hunger the subsequent morning. It is acknowledged that these changes in eating routine may be unique to student populations.

The present findings do not support the idea that the lower BMI of breakfast eaters compared with breakfast skippers are caused by increased energy expenditures in the former during the morning or because the former eat less during the day. Thus, these findings may be an indication that the known association between breakfasting frequency and $\mathrm{BMI}^{(2)}$ is a result of habitual breakfast consumption simply being a marker for appropriate dietary patterns ${ }^{(4)}$.

1. Kosti RI, Panagiotakos D, Zampelas A et al. (2008) Pub Health Nutr 11, 1015-1021.

2. Timlin MT, Pereira MA, Story M et al. (2008) Pediatrics 121, e636-e645.

3. Achten J \& Jeukendrup A (2003) Sports Med 33, 517-538.

4. Ruxton CHS \& Kirk TR (1997) Br J Nutr 78, 199-213. 